\title{
Electrical Vagus Nerve Stimulation for the Treatment of Chronic Heart Failure
}

\author{
Hani N. Sabbah, Ph.D., FACC, FCCP, FAHA \\ Department of Medicine, Division of Cardiovascular Medicine, Henry Ford Heart \& Vascular \\ Institute, Henry Ford Hospital, Detroit, Michigan
}

\begin{abstract}
Autonomic dysregulation is a feature of chronic heart failure (HF) and is characterized by a sustained increase of sympathetic drive and by withdrawal of parasympathetic activity. Both sympathetic overdrive and increased heart rate are predictors of poor long-term outcome in patients with HF. Pharmacologic agents that partially inhibit sympathetic activity such as betaadrenergic receptor blockers are effective in reducing mortality and morbidity in patients with chronic HF. In contrast, modulation of parasympathetic activation as a potential therapy for HF has received only limited attention due to its inherent complex cardiovascular effects. This review examines results of experimental animal studies that provide support for the possible use of electrical Vagus nerve stimulation (VNS) as a long-term therapy for the treatment of chronic HF. The review will also address the effects of VNS on potential modifiers of the HF state including pro-inflammatory cytokines, nitric oxide elaboration, and myocardial expression of gap junction proteins. Finally, we will briefly review the safety, feasibility and efficacy trends of VNS in patients with advanced HF.
\end{abstract}

\section{Keywords}

Ventricular function; ventricular remodeling; Vagus nerve stimulation; sympathetic activity; parasympathetic activity

\section{Introduction}

Autonomic imbalance characterized by sustained sympathetic overdrive and by parasympathetic withdrawal is a key maladaptation of the HF state. This autonomic dysregulation has long been recognized as a mediator of increased mortality and morbidity in myocardial infarction and HF $(1,2)$. Sympathovagal imbalance in HF can lead to increased heart rate, excess release of pro-inflammatory cytokines, dysregulation of nitric oxide pathways and arrythmogenesis. Diminished vagal activity reflected in increased heart rate is a predictor of high mortality in $\mathrm{HF}(3,4)$. Sustained increase of sympathetic activity contributes to progressive left ventricular (LV) dysfunction in $\mathrm{HF}$ and promotes progressive LV remodeling $(5,6)$. Pharmacologic agents that reduce heart rate such as beta-blockers and, more recently, specific and selective inhibitors of the cardiac pacemaker current I $f$ have been shown to improve survival and prevent or attenuate progressive $\mathrm{LV}$ remodeling in animals in $\operatorname{HF}(4,5,7,8)$.

Address for Correspondence, Hani N. Sabbah, PhD, Director, Cardiovascular Research, Henry Ford Hospital, 2799 West Grand Boulevard, Detroit, Michigan 48202, Office Phone: (313) 916-7360, Office Fax: (313) 916-3001, hsabbah1 @ hfhs.org. 
During the past 2-3 decades emphasis was placed almost exclusively on treating chronic HF through modulation of neurohumoral activation; an emphasis that gave rise to angiotensinconverting enzyme inhibitors, beta-adrenergic receptor blockers, and aldosterone antagonists. In recent years, renewed interest has emerged in modulating parasympathetic or vagal activity as a therapeutic target for treating chronic HF. In Alteration in cardiac vagal efferent activity through peripheral cardiac nerve stimulation can produce bradycardia and can modification atrial as well as ventricular contractile function $(9,10)$. Electrical Vagus nerve stimulation (VNS) was shown to prevent sudden cardiac death in dogs with myocardial infarction and to improve long-term survival in rats with chronic $\operatorname{HF}(11,12)$. VNS has also been shown to suppress arrhythmias in conscious rats with chronic HF secondary to myocardial infarction (13). The balance of this review will focus primarily on the effects of chronic VNS on LV dysfunction and remodeling in dogs with HF produced by multiple sequential intracoronary microembolizations (14) or by high-rate ventricular pacing (15) and on the safety, feasibility and efficacy trends of VNS in patients with advanced HF (16).

The CardioFit ${ }^{\circ}$ VNS system (BioControl Medical Ltd, Yehud, Israel) used in dogs with coronary microembolization-induced HF delivered electrical stimulation to the right cervical Vagus only when heart rate increased beyond a pre-set level thus operating on a negativefeedback loop. The stimulation Lead (CSL) is a modified bi-polar cuff electrode designed for VNS. The lead activates vagus cardioinhibitory B fibers while maintaining a large degree of unidirectionality with respect to low threshold fibers recruited in the 1-2 mA range. The lead is attached to a model 5000 electrostimulator. The stimulator or generator was fitted with a processing unit that adjusted the impulse rate and intensity to keep the heart rate within the desired range. Maximal stimulation current, pulse width and operation algorithm are controlled by the Physician Programmer via wireless communication. A standard pacemaker bi-polar ventricular electrode was used in all the discussed animal studies for sensing the intracardiac electrocardiogram. A model 5300 BioControl physician programmer was used in conjunction with the nerve stimulator to non-invasively adjust the stimulation algorithm and settings for optimal outcome of therapy in each individual case. Adjustments were made while the programming wand is placed over the implanted nerve stimulator.

\section{VNS in Dogs with Microembolization-Induced HF}

The CardioFit VNS system (BioControl Medical Ltd, Yehud, Israel) used in dogs with coronary microembolization-induced HF delivered electrical stimulation to the right cervical Vagus only when the heart rate increased beyond a pre-set level thus operating on a negative-feedback loop. The stimulation lead was a modified bi-polar cuff electrode designed to activate vagus cardioinhibitory $\mathrm{B}$ fibers while maintaining a large degree of unidirectionality with respect to low threshold fibers recruited in the 1-2 mA range. The lead is attached to a model 5000 electrostimulator fitted with a processing unit that adjusts the impulse rate to keep the heart rate within the desired range. Maximal stimulation current, pulse width and operation algorithm are controlled by the Physician Programmer via wireless communication. A standard pacemaker bi-polar ventricular electrode also attached to the model 5000 electrostimulator, was used in all the animal studies for sensing the intracardiac electrocardiogram.

\section{Monotherapy With VNS}

Dogs with HF and LV ejection fraction of approximately $35 \%$ were randomized to 3 months of active VNS monotherapy (CardioFit ON, $n=7$ ) or to no therapy at all (Sham-operated control, CardioFit OFF, $\mathrm{n}=6$ ). The feedback on-demand heart rate control was set to reduce basal heart rate by $10 \%$ (17). Long-term (3 months) VNS monotherapy significantly 
improved LV ejection fraction significantly decreased LV end-systolic and end-diastolic volumes compared to controls (Table 1) (17). The reduction in LV size was in-line with an observed decrease in plasma levels of n-terminal pro-brain natriuretic peptide (nt-pro BNP) (Table 1). In VNS-treated dogs, heart rate assessed using ambulatory ECG Holter monitoring showed a reduction of minimum, average and maximum heart rate by 1,10 , and 28 beats/min respectively compared to changes of heart rate in control dogs of 2,1 and 0.5 beats/min respectively.

Long-term VNS therapy also elicited improvements in indices of LV diastolic function. VNS significantly decreased LV end-diastolic pressure (Table 1), increased deceleration time of rapid mitral inflow velocity, tended to increase the ratio of peak mitral inflow velocity during early LV filling to peak mitral inflow velocity during left atrial contraction (PE/PA) and significantly reduced LV end-diastolic circumferential wall stress, a determinant of myocardial oxygen consumption (Table 1). These measures suggest that VNS can reduce preload, improve LV relaxation and improve LV function without increasing myocardial oxygen consumption.

\section{VNS in Combination with Beta-Blockade}

The effects of VNS stimulation in dogs with HF was also examined on top of background therapy with beta-blockade. Dogs with LV ejection fraction $\sim 35 \%$ were randomized to 3 months therapy with a beta-blocker alone (metoprolol succinate, $100 \mathrm{mg}$ once daily, $\mathrm{n}=6$ ) or to metoprolol (100 mg once daily) combined with active VNS with CardioFit ( $\mathrm{n}=6)$. As with the monotherapy study, the CardioFit VNS system was operated in the feedback on-demand heart rate responsive mode. Dogs were started on oral metoprolol therapy 2 weeks prior to randomization to VNS therapy. After randomization, all dogs continued to receive metoprolol succinate once daily for the entire duration of the study (18).

In HF dogs receiving background therapy with metoprolol, the addition of VNS increased LV ejection fraction and decreased LV end-systolic volume compared to dogs treated with metoprolol alone (Table 2) (18). These findings suggest that the addition of VNS improves LV systolic function beyond that seen with beta-blockade alone. Adding VNS therapy to metoprolol also elicited improvements in indices of $\mathrm{LV}$ diastolic function. Combination therapy resulted in a greater lowering LV end-diastolic pressure and LV end-diastolic wall stress and a greater increase of deceleration time of rapid mitral inflow velocity compared to meptoprolol alone (Table 2).

The improvements in LV systolic and diastolic function with combination therapy were associated with important changes of heart rate. Twenty four hour ambulatory ECG Holter monitoring studies showed no differences in minimum and average heart rate between dogs treated with metoprolol and those treated with combination therapy with VNS. Maximum heart rate, however, was significantly lower in dogs treated with the combination therapy $(114 \pm 12$ vs. $149 \pm 8$ beats/min, $\mathrm{p}<0.05)$. These observations suggest that preventing heart rate escape at the high end and in doing so, reduce the adverse impact of periods of increased cardiac workload and increased myocardial oxygen consumption in dogs treated with VNS in combination with beta-blockade may elicit benefits in the form of improved LV systolic function compared to beta-blockade alone.

\section{VNS and LV Remodeling}

In addition to improving LV systolic and diastolic function, long-term VNS in dogs with coronary microembolization-induced HF elicited important changes in cellular and structural markers of LV remodeling. Compared to untreated HF dogs, dogs treated with VNS as monotherapy showed a significant decrease of volume fraction of replacement and 
interstitial fibrosis, a decrease of oxygen diffusion distance, measured as half the distance between 2 adjoining capillaries, and a decrease of myocyte cross-sectional area, a measure of cardiomyocyte hypertrophy, and an increase in capillary density (Table 3). The above histomorphometric measures are often if not always adversely affected by the HF state. Amelioration of these structural measures by VNS suggests that this form of therapy can help preserve myocardial structural integrity through direct or indirect action on the failing myocardium.

\section{VNS and Pro-Inflammatory Cytokines, Nitric Oxide and Gap Junction Proteins}

Elevation of pro-inflammatory cytokines occurs in HF and is associated with increased morbidity and mortality. Electrical VNS has been shown to decrease the release of various cytokines including tumor necrosis factor-alpha (TNF-a) and interlukin-6 (IL-6) (20). In dogs with microembolization-induced HF, LV tissue levels of TNF- $\alpha$ and IL-6 are elevated compared to LV tissue from normal dogs (Table 3). Long-term monotherapy with VNS normalizes protein expression of both TNF-a and IL-6 in LV myocardium (Table 3) (21).

Nitric oxide (NO) is formed by a family of nitric oxide synthases (NOS). The 3 isoforms of NOS identified to date are endothelin NOS (eNOS), inducible NOS (iNOS) and neuronal NOS (nNOS). Nitric oxide produced by eNOS plays an important role in the regulation of cell growth and apoptosis (22) and can enhance myocardial relaxation and regulate contractility $(22,23)$. In dogs with coronary microembolization-induced HF, mRNA and protein expression of eNOS in LV myocardium is significantly down-regulated compared to normal dogs and therapy with VNS significantly improves the expression of eNOS (24) (Table 3). Inducible NOS is up-regulated in HF. Overexpression of iNOS in cardiomyocytes in mice results in peroxynitrite generation associated with fibrosis, LV hypertrophy, chamber dilation, cardiomyopathic phenotype, heart block and sudden cardiac death (25). In dogs with coronary microembolization-induced HF, mRNA and protein expression of iNOS in LV myocardium is significantly up-regulated compared to normal dogs and long-term therapy with VNS tends to normalize the expression of iNOS (24) (Table 3). Neuronal NOS has been shown to be up-regulated in rats following myocardial infarctions and in the human failing heart (26). In rats with HF, inhibition of nNOS leads to increased sensitivity of the myocardium to beta-adrenergic stimulation (27). This observation suggests a role for $\mathrm{nNOS}$ in the autocrine regulation of myocardial contractility (27). In dogs with coronary microembolization-induced HF, mRNA and protein expression of nNOS in LV myocardium was shown to be significantly up-regulated compared to normal dogs and long-term therapy with VNS tends to normalize the expression of nNOS in the failing dog LV myocardium (24) (Table 3).

Gap junction proteins or connexins are reduced or redistributed from intercalated disks to lateral cell borders in a variety of cardiac diseases including HF (28). This so-called "gap junction remodeling" is considered highly arrhythmogenic. In mammals, gap junctions exclusively contain connexin-43 (Cx43). Reduced expression of $\mathrm{Cx} 43$ occurs in the failing human heart and has been shown to result in slowed transmural conduction and dispersion of action potential duration with increased susceptibility to arrhythmia and sudden cardiac death $(29,30)$. In dogs with coronary microembolization-induced HF, mRNA and protein expression of $\mathrm{Cx} 43$ in LV myocardium was shown to be markedly down-regulated compared to normal dogs and long-term therapy with VNS was associated with a significant increase in the expression of Cx43 in LV myocardium of dogs with HF (31) (Table 3).

\section{VNS in Dogs with Rapid Pacing-Induced Heart Failure}

Electrical VNS as a potential therapy for HF was also recently examined in dogs with HF secondary to high-rate ventricular pacing using the Cyberonics VNS system (Cyberonics 
Inc., Houston, TX) which did not operate on a negative feedback mechanism (15). In this study, VNS therapy was delivered continuously for the duration of the study with a duty cycle of 14 seconds ON and 12 seconds OFF. VNS signals were delivered to the right cervical vagus nerve at a frequency of $20 \mathrm{~Hz}$ and a pulse width of $0.5 \mathrm{~ms}$ (15). Dogs were randomized to control $(n=7)$ or to monotherapy with VNS $(n=8)$ and followed for 8 weeks. All measurements were made approximately $15 \mathrm{~min}$ after temporarily turning off the ventricular pacemaker and the Vagus nerve stimulator (15). VNS therapy resulted in a significant decrease of LV end-diastolic and end-systolic volumes and a significant increase of LV ejection fraction compared to controls (15). This improvement was associated with significant reduction in plasma levels of norepinephrine, angiotensin-II and c-reactive protein. The study also demonstrated the effectiveness of VNS in restoring baroreflex sensitivity thus improving cardiac autonomic control (15). Because rapid pacing was maintained throughout the study except for short periods of time when measurements were made, one can argue that the benefits of VNS therapy, in this model of HF, are independent of heart rate (15).

\section{Safety and Tolerability of VNS in Patients with Advanced HF}

In patients with HF, reduced vagal activity is associated with increased mortality (1). Further vagal withdrawal has also been shown to precede episodes of acute decompensation (32). In a recently published study, De Ferrari et al. on behalf of the CardioFit Multicenter Trial Investigators, examined the safety and tolerability of chronic VNS in 32 patients with symptomatic HF and severe LV dysfunction using the CardioFit system (16). The CardioFit system used in this study was different than that used in dogs with microembolizationinduced HF in that it did not operate on a negative feedback principle. A bradycardia limit causing interruption of VNS was set at 55 beats/min. A 3 week up-titration period was used to maximize current amplitude and duty cycle based upon the patient sensation. The intensity of the stimulation reached $4.1 \pm 1.2 \mathrm{~mA}$ at the end of the titration period (16). The results of this multicenter, open label phase II trial with a 3-6 months follow-up with an optional 1 year follow-up suggested that VNS in HF patients with severe LV dysfunction may be safe and tolerable. Trends for efficacy were also favorable bearing in mind the nonrandomized and unblinded nature of the study design. The study showed significant improvements in NYHA class, 6 minute walk test, LV ejection fraction and LV systolic volumes (16).

\section{Conclusions}

A wealth of pre-clinical and clinical studies support of the concept of electrical VNS can favorably modify the underlying pathophysiology and course of evolving HF. In animals with HF, VNS improves LV function, attenuates LV remodeling and may prevent arrhythmias that provoke sudden cardiac death. VNS derives these potential clinical benefits from multiple mechanisms of action that include reduced heart rate and normalization of sympathetic over-drive. VNS also appears to have a favorable impact on other signaling pathways that are likely to elicit beneficial effects in patients with HF. These include restoration of baroreflex sensitivity, suppression of pro-inflammatory cytokines, normalization of nitric oxide signaling pathways and suppression of gap junction remodeling. At present, there is no evidence to implicate a single mechanism of action for the benefits derived from VNS. Instead, it is most likely that all of the mechanisms listed above act in concert to elicit the global benefit seen with VNS. In humans with heart failure, VNS may be safe, feasible and appears to be well tolerated. Full appreciation for the efficacy of VNS in treating chronic HF must await completion of pivotal randomized clinical trials. 


\section{Acknowledgments}

Supported, in part, by research grants from BioControl Medical, Ltd. and National Heart, Lung, and Blood Institute PO1 HL074237-06

\section{References}

1. Schwatrz PJ, Vanoli E, Stramba-Badiale M, De Ferrari GM, Billman GE, Foreman RD. Autonomic mechanisms and sudden death. New insights from the analysis of baroreceptor reflexes in conscious dogs with and without myocardial infarction. Circulation. 1988; 78:969-973. [PubMed: 3168199]

2. Mortara A, La Rovere MT, Pinna GD, Prpa A, Maestri R, Febo O, Pozzoli M, Opasich C, Tavazzi L. Arterial baroreflex modulation of heart rate in chronic heart failure: clinical and hemodynamic correlates and prognostic implications. Circulation. 1997; 96:3450-3458. [PubMed: 9396441]

3. La Rovere MT, Bigger JT, Marcus FI, Mortara A, Schwartz PJ. Baroreflex sensitivity and heart-rate variability in prediction of total cardiac mortality after myocardial infarction. Lancet. 1998; 351:478-484. [PubMed: 9482439]

4. Lechat P, Hulot JS, Escolano S, Mallet A, Leizorovicz A, Werhlen-Grandjean M, Pochmalicki G, Dargie H. on behalf of the CIBIS II Investigators. Heart rate and cardiac rhythm relationships with bisoprolol benefit in chronic heart failure in CIBIS II trial. Circulation. 2001; 103:1428-1433. [PubMed: 11245648]

5. Sabbah HN, Shimoyama H, Kono T, Gupta RS, Sharov VG, Scicli G, Levine TB, Goldstein S. Effects of long-term monotherapy with enalapril, metoprolol and digoxin on the progression of left ventricular dysfunction and dilation in dogs with reduced ejection fraction. Circulation. 1994; 89:2852-2859. [PubMed: 8205701]

6. Sabbah HN, Stanley WC, Sharov VG, Mishima T, Tanimura M, Benedict CR, Hegde S, Goldstein $\mathrm{S}$. Effects of dopamine $\beta$-hydroxylase inhibition with nepicastat on the progression of left ventricular dysfunction and remodeling in dogs with chronic heart failure. Circulation. 2000; 102:1990-1995. [PubMed: 11034950]

7. Cheng Y, George I, Yi G-H, Reiken S, Gu A, Tao YK, Muraskin J, Qin S, He K-L, Hay I, Yu K, Oz MC, Burkhoff D, Holmes J, Wang J. Bradycardic therapy improves left ventricular function and remodeling in dogs with coronary embolization-induced chronic heart failure. J Pharmacol Experiment Therap. 2007:469-476. 2007.

8. Swedberg K, Komajda M, Bohm M, Borer JS, Ford I, Dubost-Brama A, Lerebours G, Tavazzi L. on behalf of the SHIFT Investigators. Ivabradine and outcomes in chronic heart failure (SHIFT): a randomised placebo-controlled study. Lancet. 2010; 376:875-885. [PubMed: 20801500]

9. Kunze DL. Reflex discharge patterns of cardiac vagal efferent fibers. J Physiol (Lond). 1972; 222:1-8. [PubMed: 4338691]

10. Harman MA, Reeves TJ. Effects of efferent nerve stimulation on atrial and ventricular function. Am J Physiol. 1968; 215:1210-1217. [PubMed: 5687515]

11. Vanoli E, De Ferrari GM, Stramba-Badiale M, Hull SS Jr, Foreman RD, Schwartz PJ. Vagal stimulation and prevention of sudden death in conscious dogs with healed myocardial infarction. Circ Res. 1991; 68:1471-1481. [PubMed: 2019002]

12. Li M, Zheng C, Sato T, Kawada T, Sugimachi N, Sunagawa K. Vagal nerve stimulation markedly improves long-term survival after chronic heart failure in rats. Circulation. 2004; 109:120-124. [PubMed: 14662714]

13. Zheng C, Li M, Inagaki M, Kawada T, Sunagawa K, Sugimachi M. Vagal stimulation markedly suppresses arrhythmias in conscious rats with chronic heart failure after myocardial infarction. Conf. Proc IEEE Eng Med Biol Soc. 2005; 7:7072-7075. [PubMed: 17281904]

14. Sabbah HN, Stein PD, Kono T, Gheorghiade M, Levine TB, Jafri S, Hawkins ET, Goldstein S. A canine model of chronic heart failure produced by multiple sequential coronary microembolizations. Am J Physiol. 1991; 260:H1379-H1384. [PubMed: 1826414]

15. Zhang Y, Popovic ZB, Bibevski S, Fakhry I, Sica DA, Van Wagoner DR, Mazgalev TN. Chronic vagus nerve stimulation improves autonomic control and attenuates systemic inflammation and heart failure progression in a canine high-rate pacing model. Circulation. 2009; 2:692-699. [PubMed: 19919995] 
16. De Ferrari GM, Crijns HJGM, Borggrefe M, Milasinovic G, Smid J, Zabel M, Gavazzi A, Sanzo A, Dennert R, Kuschyk J, Raspopovic S, Klein H, Swedberg K, Schwartz PJ. Chronic vagus nerve stimulation: a new and promising therapeutic approach for chronic heart failure. Eur Heart J. 2010 Nov.12 [Epub ahead of print].

17. Sabbah HN, Rastogi S, Mishra S, Gupta RC, Ilsar I, Imai M, Cohen U, Ben-David T, Ben-Ezra O. Long-term therapy with neuroselective electric Vagus nerve stimulation improves LV function and attenuates global LV remodelling in dogs with chronic heart failure. Europ J Heart Failure Supplements. 2005; 4:166. (Abstract).

18. Sabbah HN, Imai M, Zaretsky A, Rastogi S, Wang M, Jiang A, Zaca V. Therapy with Vagus nerve electrical stimulation combined with beta-blockade improves left ventricular function in dogs with heart failure beyond that seen with beta-blockade alone (Abstract). Europ J Heart Failure. 2007; 6:114.

19. Liu YH, Yang XP, Sharov VG, Nass O, Sabbah HN, Peterson E, Carretero OA. Effects of angiotensin-converting enzyme inhibitors and angiotensin ii type 1 receptor antagonists in rats with heart failure. Role of kinins and angiotensin ii type 2 receptors. J Clin Invest. 1997; 99:19261935. [PubMed: 9109437]

20. Wang H, Yu M, Ochani M, Amella CA, Tanovic M, Susarla S, Li JH, Wang H, Yang H, Ulloa L, Czura CJ, Tracey KJ. Nicotinic acetylcholine receptor alpha7 subunit is an essential regulator of inflammation. Nature. 2003; 421:384-388. [PubMed: 12508119]

21. Gupta RC, Imai M, Jiang AJ, Wang M, Sabbah HN. Chronic therapy with selective electric vagus nerve stimulation normalizes plasma concentration of tissue necrosis factor- $\alpha$, interleukin- 6 and Btype natriuretic peptide in dogs with heart failure (Abstract). J Am Coll Cardiol. 2006; 47:77A.

22. Feng Q, Song W, Lu X, Hamilton JA, Lei M, Peng T, Yee S-P. Development of Heart Failure and congenital septal defects in mice lacking endothelial nitric oxide synthase. Circulation. 2002; 106:873-879. [PubMed: 12176963]

23. Kelly RA, Balligand JL, Smith TW. Nitric oxide and cardiac function. Circ Res. 1996; 79:363380. [PubMed: 8781470]

24. Gupta RC, Mishra S, Rastogi S, Imai M, Zaca V, Sabbah HN. Chronic therapy with electric Vagus Nerve stimulation normalizes mRNA and protein expression of nitric oxide synthase in myocardium of dogs with heart failure (Abstract). Europ Heart J. 2006; 27:477.

25. Mungrue IN, Gros R, You X, Pirani A, Azad A, Csont T, Schulz R, Butany J, Stewart DJ, Husain M. Cardiomyocyte overexpression of iNOS in mice results in peroxynitrite generation, heart block and sudden death. J Clin Invest. 2002; 109:735-743. [PubMed: 11901182]

26. Damy T, Ratajczak P, Shah AM, Camors E, Marty I, Hasenfuss G, Marotte F, Samuel JL, Heymes C. Increased neuronal nitric oxide synthase-derived NO production in the failing human heart. Lancet. 2004; 363:1365-1367. [PubMed: 15110495]

27. Bendall JK, Damy T, Ratajczak P, Loyer X, Monceau V, Marty I, Milliez P, Robidel E, Marotte F, Samuel J-L, Heymes C. Role of myocardial neuronal nitric oxide synthase-derived nitric oxide in $\beta$-adrenergic hyporesponsiveness after myocardial infarction-induced heart failure in rat. Circulation. 2004; 110:2368-2375. [PubMed: 15466641]

28. Severs NJ, Bruce AF, Dupont E, Rothery S. Remodelling of gap junctions and connexin expression in diseased myocardium. Cardiovasc Res. 2008; 80:9-19. [PubMed: 18519446]

29. Wang XJ, Gerdes AM. Chronic pressure overload cardiac hypertrophy and failure in guinea pigs: III. Intercalated disc remodeling. J Mol Cell Cardiol. 1999; 31:333-343. [PubMed: 10093046]

30. Ai X, Pogwizd M. Connexin 43 downregulation and dephosphorylation in nonischemic heart failure is associated with enhanced colocalized protein phosphatase type 2A. Circ Res. 2005; 96:54-63. [PubMed: 15576650]

31. Rastogi S, Mishra S, Ilsar I, Zaretsky A, Sabbah HN. Chronic therapy with electric Vagus nerve stimulation normalizes mRNA and protein expression of connexin-40, -43 and -45 in left ventricular myocardium of dogs with heart failure (Abstract). Circulation. 2007; 116:II-218.

32. Adamson PB, Smith AL, Abraham WT, Kleckner KJ, Stadler RW, Shih A, Rhodes MM. InSync III Model 8042 and Attain OTW Lead Model 4193 Clinical Trial Investigators. Continuous autonomic assessment in patients with symptomatic heart failure: prognostic value of heart rate 
variability measured by an implanted cardiac resynchronization device. Circulation. 2004; 110:2389-2394. [PubMed: 15313946] 


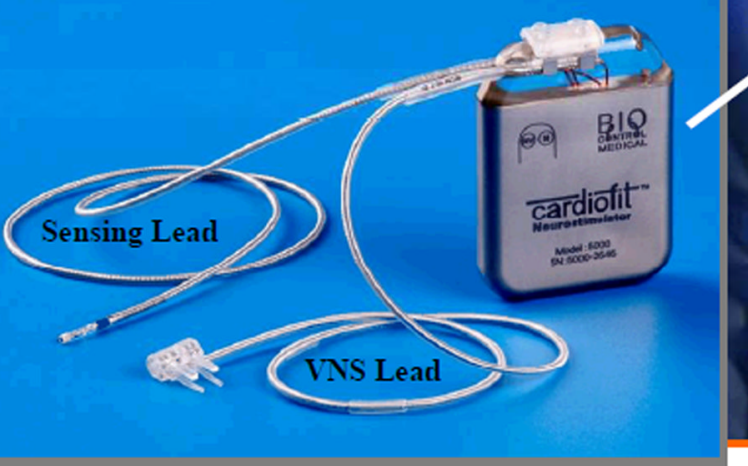

Figure 1.

Right: Depiction of an implanted CardioFit Vagus Nerve Stimulation (VNS) device showing the position of the VNS lead on the right vagus nerve, the intracardiac pacing lead in the right ventricular apex and the implantable CardioFit neurostimulator in the right subclavicular region. Left Top: Depiction of the positioning of the CardioFit stimulation lead around the right vagus nerve. Left Bottom: Photograph of the Cardiofit VNS implantable neurostimulator, sensing lead and VNS lead. 

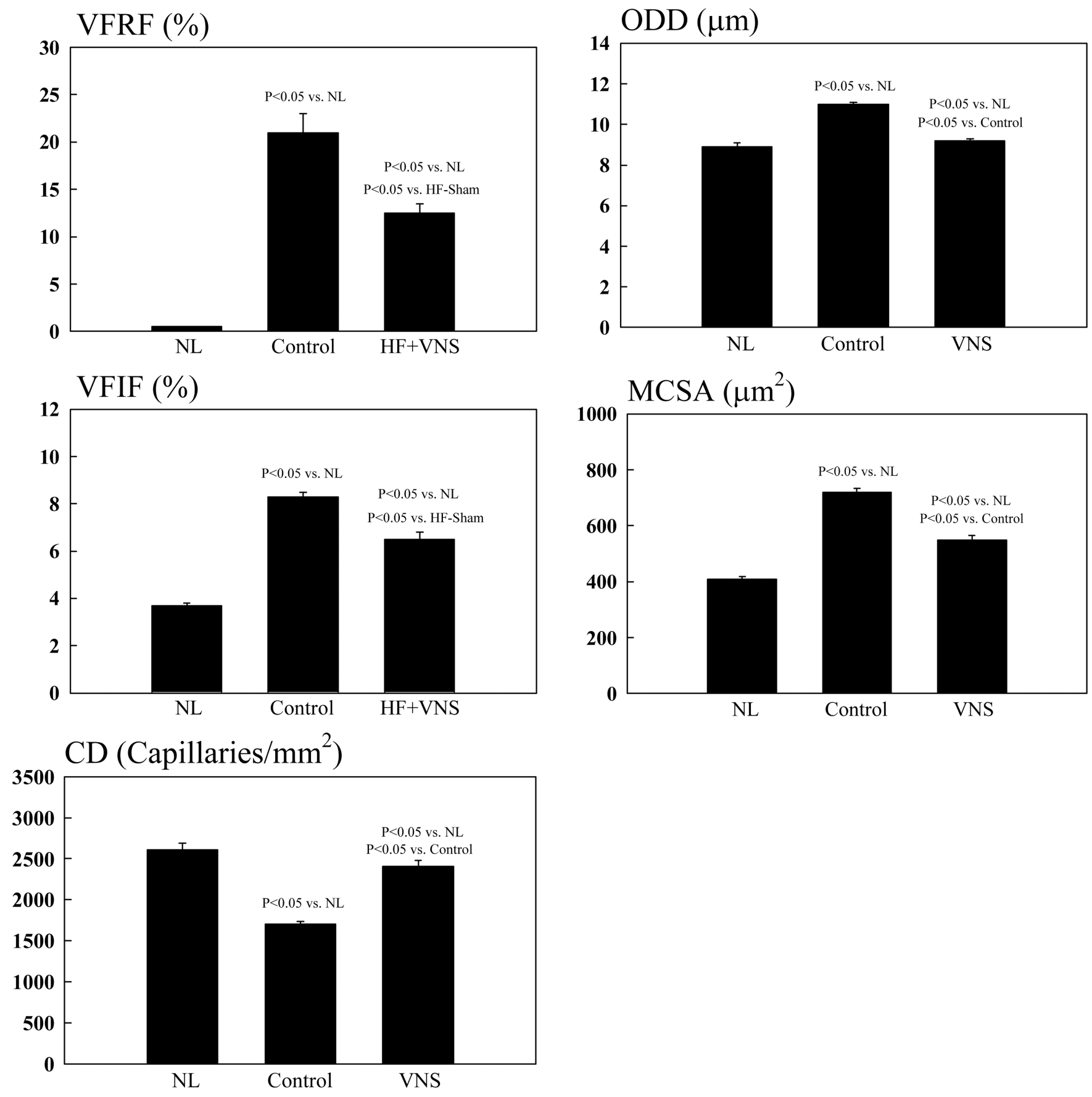

Figure 2.

Bar graphs (mean \pm standard error) illustrating the changes in volume fraction of replacement fibrosis (VFRF), volume fraction of interstitial fibrosis (VFIF), capillary density (CD), oxygen diffusion distance (ODD) and myocyte cross-sectional area (MCSA) between normal dogs (NL), untreated heart failure dogs (Control) and heart failure dogs treated with vagus nerve stimulation (VNS). 
Table 1

Indices of left ventricular systolic and diastolic function in control dogs and VNS-treated dogs obtained prior to initiating therapy (PRE) and at 3 months after initiating therapy (POST).

\begin{tabular}{|l|c|c|c|c|}
\hline \multirow{2}{*}{} & \multicolumn{2}{|c|}{ CONTROL (n=6) } & \multicolumn{2}{c|}{ VNS (n=7) } \\
\cline { 2 - 5 } & PRE & POST & PRE & POST \\
\hline LV EF (\%) & $31 \pm 1$ & $29 \pm 1^{*}$ & $34 \pm 1$ & $41 \pm 2^{*}$ \\
\hline LV EDV (ml) & $56 \pm 2$ & $60 \pm 2^{*}$ & $58 \pm 4$ & $57 \pm 4^{*}$ \\
\hline LV ESV (ml) & $37 \pm 1$ & $42 \pm 2^{*}$ & $38 \pm 2$ & $34 \pm 3^{*}$ \\
\hline LVEDP $(\mathbf{m m H g})$ & $14 \pm 1$ & $15 \pm 1$ & $15 \pm 1$ & $11 \pm 2^{*}$ \\
\hline PE/PA & $2.0 \pm 0.2$ & $1.8 \pm 0.2$ & $2.0 \pm 0.3$ & $2.4 \pm 0.4$ \\
\hline DT $(\mathbf{m s e c})$ & $91 \pm 1$ & $85 \pm 4$ & $94 \pm 5$ & $111 \pm 10^{*}$ \\
\hline EDWS $\left(\mathbf{g m} / \mathbf{c m}^{\mathbf{2}}\right)$ & $54 \pm 3$ & $57 \pm 4$ & $60 \pm 5$ & $46 \pm 7^{*}$ \\
\hline
\end{tabular}

VNS=Vagus nerve stimulation; $\mathrm{LV}=$ left ventricular; $\mathrm{EF}=$ ejection fraction; $\mathrm{EDV}=$ end-diastolic volume; $\mathrm{ESV}=\mathrm{end}$-systolic volume; nt-pro $\mathrm{BNP}=\mathrm{n}$ terminal pro brain natriuretic peptide; LVEDP=left ventricular end-diastolic pressure; $\mathrm{PE} / \mathrm{PA}=$ ratio of peak early rapid mitral inflow velocity (PE) to peak of mitral inflow velocity during left atrial contraction (PA); DT=deceleration time of early rapid mitral inflow velocity; EDWS=left ventricular end-diastolic circumferential wall stress.

$=\mathrm{p}<0.05$ vs. PRE. 
Table 2

Indices of left ventricular systolic and diastolic function obtained prior to initiating therapy (PRE) and at 3 months after initiating therapy (POST) in heart failure dogs treated with a beta-blocker alone and dogs treated with beta-blocker in combination with VNS therapy

\begin{tabular}{|c|c|c|c|c|}
\hline & \multicolumn{2}{|c|}{$\beta$-Blocker } & \multicolumn{2}{|c|}{ ß-Blocker + VNS } \\
\hline & PRE & POST & PRE & POST \\
\hline LV EF (\%) & $31 \pm 1$ & $36 \pm 1^{*}$ & $33 \pm 1$ & $43 \pm 1^{*}$ \\
\hline LV EDV (ml) & $56 \pm 4$ & $56 \pm 3$ & $56 \pm 2$ & $56 \pm 2$ \\
\hline LV ESV (ml) & $39 \pm 3$ & $36 \pm 2$ & $37 \pm 1$ & $32 \pm 1^{*}$ \\
\hline LV EDP (mmHg) & $13.3 \pm 0.8$ & $10.5 \pm 0.6^{*}$ & $12.7 \pm 0.8$ & $8.5 \pm 0.7^{*}$ \\
\hline $\mathbf{P E} / \mathbf{P A}$ & $1.7 \pm 0.1$ & $2.1 \pm 0.2$ & $1.5 \pm 0.1$ & $2.1 \pm 0.1$ * \\
\hline DT (msec) & $80.2 \pm 1.3$ & $86.0 \pm 1.3^{*}$ & $78.3 \pm 1.3$ & $91.3 \pm 1.5^{*}$ \\
\hline $\operatorname{EDWS}\left(\mathrm{gm} / \mathrm{cm}^{2}\right)$ & $51.5 \pm 3$ & $40.0 \pm 2.0$ & $45.9 \pm 4.0$ & $29.6 \pm 3.0^{*}$ \\
\hline
\end{tabular}

Abbreviations as in Table 1.

$=\mathrm{p}<0.05$ vs. PRE. 
Table 3

mRNA expression of pro-inflammatory cytokines, nitric oxide synthases and connexin- 43 in LV myocardium of normal (NL) dogs, untreated heart failure dogs (Control) and dogs with heart failure treated with vagus nerve stimulation (VNS)

\begin{tabular}{|l|c|c|c|}
\hline & NL $(\mathbf{n = 6})$ & CONTROL $(\mathbf{n = 6})$ & VNS $(\mathbf{n = 7})$ \\
\hline TNF-a (du) & $173 \pm 14$ & $399 \pm 12^{*}$ & $202 \pm 21^{\dagger}$ \\
\hline IL-6 (du) & $75 \pm 9$ & $246 \pm 23^{*}$ & $116 \pm 19^{\dagger}$ \\
\hline eNOS (du) & $144 \pm 15$ & $51 \pm 2^{*}$ & $90 \pm 3^{\dagger}$ \\
\hline iNOS (du) & $168 \pm 15$ & $405 \pm 14^{*}$ & $269 \pm 28^{\dagger}$ \\
\hline nNOS (du) & $155 \pm 11$ & $441 \pm 52^{*}$ & $198 \pm 20^{\dagger}$ \\
\hline Cx43 (du) & $218 \pm 16$ & $11 \pm 2^{*}$ & $106 \pm 5^{\dagger}$ \\
\hline
\end{tabular}

TNF- $\mathrm{a}=$ tumor necrosis factor-alpha; IL-6=interlukin-6; eNOS=endothelial nitric oxide synthase; iNOS=inducible nitric oxide synthase; nNOS=neuronal nitric oxide synthase; $\mathrm{Cx} 43=$ connexin- 43 ; du=densitometric units.

$$
\begin{aligned}
& *=p<0.05 \text { vs. } \mathrm{NL} ; \\
& { }^{*}=\mathrm{p}<0.05 \text { vs. CONTROL. }
\end{aligned}
$$

